\title{
Approach for Selection of Rayleigh Damping Parameters Used for Time History Analysis
}

\section{ASME Pressure Vessels and Piping Division Conference}

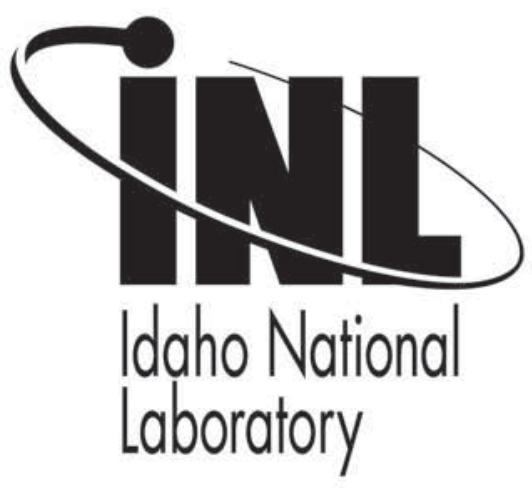

This is a preprint of a paper intended for publication in a journal or proceedings. Since changes may be made before publication, this preprint should not be cited or reproduced without permission of the author. This document was prepared as an account of work sponsored by an agency of the United States Government. Neither the United States Government nor any agency thereof, or any of their employees, makes any warranty, expressed or implied, or assumes any legal liability or responsibility for any third party's use, or the results of such use, of any information, apparatus, product or process disclosed in this report, or represents that its use by such third party would not infringe privately owned rights. The views expressed in this paper are not necessarily those of the United States Government or the sponsoring agency. 


\section{APPROACH FOR SELECTION OF RAYLEIGH DAMPING PARAMETERS USED FOR TIME HISTORY ANALYSIS}

\author{
R. E. Spears, Ph.D. \\ Senior Research Engineer \\ Idaho National Laboratory \\ Tel: 208-526-4109 \\ Fax: 208-526-4311 \\ E-Mail: Robert.Spears@inl.gov
}

\author{
S. R. Jensen \\ Senior Analysis Integrator \\ Idaho National Laboratory \\ Tel: 208-526-9137 \\ Fax: 208-526-9259 \\ E-Mail: Stuart.Jensen@inl.gov
}

\begin{abstract}
Nonlinearities, whether geometric or material, need to be addressed in seismic analysis. One good analysis method that can address these nonlinearities is direct time integration with Rayleigh damping. Modal damping is the damping typically specified in seismic analysis Codes and Standards [1,2]. Modal damping is constant for all frequencies where Rayleigh damping varies with frequency. An approach is proposed here for selection of Rayleigh damping coefficients to be used in seismic analyses that are consistent with given Modal damping. The approach uses the difference between the modal damping response and the Rayleigh damping response along with effective mass properties of the model being evaluated to match overall system response levels. This paper provides a simple example problem to demonstrate the approach. It also provides results for a finite element model representing an existing piping system. Displacement, acceleration, and stress results are compared from model runs using modal damping and model runs using Rayleigh damping with coefficients selected using the proposed method.
\end{abstract}

\section{INTRODUCTION}

Rayleigh damping follows the equation

$$
\zeta=\frac{\alpha}{2 \cdot \omega}+\frac{\beta \cdot \omega}{2}
$$

where the damping value " $\zeta$ " is a function of the natural frequency " $\omega$ " and the Rayleigh damping coefficients " $\alpha$ " and " $\beta$ ". This produces a curve that can be modified to match a modal damping value at one or two natural frequency points. Consequently, if a structure has one or two very dominant frequencies, Rayleigh damping can closely approximate the behavior of a prescribed modal damping. However, for more complicated structures with many modes over a large range of natural frequencies, Rayleigh damping can cause significant variation in response as compared to modal damping. A conservative approach would be to enforce a Rayleigh damping curve that matches a prescribed modal damping for the highest and lowest modes of the structure. This, however, can result in unreasonably conservative response for intermediate modes. The procedure proposed in this paper is intended to define a Rayleigh damping curve that minimizes the variation in the response as compared to modal damping. Consequently, some response that is not conservative is considered acceptable. Ultimately, the analyst must ensure that the resulting Rayleigh damping curve is reasonable for the given problem.

\section{PROCEDURE}

The proposed procedure is performed in several steps. First a plot of cumulative effective mass versus frequency is prepared from a modal analysis of the structure. If the structure model is nonlinear, it must be linearized for this step. Next, a modal damped acceleration response spectrum is generated from the time history to be used for analysis. Then, the minimum natural frequency where the Rayleigh damping curve equals the prescribed modal damping is defined. This natural frequency occurs where the cumulative effective mass plot reaches approximately $5 \%$ of the total model mass. This ensures minimal underestimation of response in the low frequency range.

The values up to this point remain unchanged. The remainder of the evaluation is iterative. First, the maximum natural frequency where the Rayleigh damping curve equals the prescribed modal damping is approximated. This initial value is taken as the frequency where the cumulative effect mass is approximately $50 \%$. Next, the Rayleigh damping coefficients 1

The United States Government retains, and by accepting the article for publication, the publisher acknowledges that the United States Government retains, a non-exclusive, paid-up, irrevocable, worldwide license to publish or reproduce the published form of this work, or allow others to do so, for United States Government purposes. 
are calculated that enforce the locations where modal damping is approximated. Also, a Rayleigh damped acceleration response spectrum is generated from the time history to be used for analysis. For each effective mass point, the modal damped response is subtracted from the Rayleigh damped response and the difference is multiplied by the effective mass value. All of these values are then summed. If the result is negative, than the Rayleigh damping coefficients are unacceptable and the selected maximum natural frequency needs to be increased. If the result is positive, then the Rayleigh damping coefficients are conservative and the selected maximum natural frequency can be decreased (as long as the result remains positive after the decrease is evaluated). If change in maximum natural frequency is needed or desired, an adjustment can be made and another iteration can be performed. The final desired result is a slightly positive value. With this result, optimization of the Rayleigh damping coefficients has been achieved.

\section{EXAMPLE PROBLEM}

For an example problem, it is desired to establish Rayleigh damping coefficients " $\alpha$ " and " $\beta$ " for the example shear frame shown in Figure 1. The example shear frame has three degrees of freedom whose displacements are identified with " $\mathrm{x}_{1}$ ", " $\mathrm{x}_{2}$ ", and " $\mathrm{x}_{3}$ ". It has an assigned modal damping value " $\zeta$ " of $5 \%$, the mass and stiffness values given below, and it is to be evaluated with the example earthquake ground motion (shown in Figure 2). (Note: This example problem is performed with Mathcad [3] using Mathcad [3] notation.)

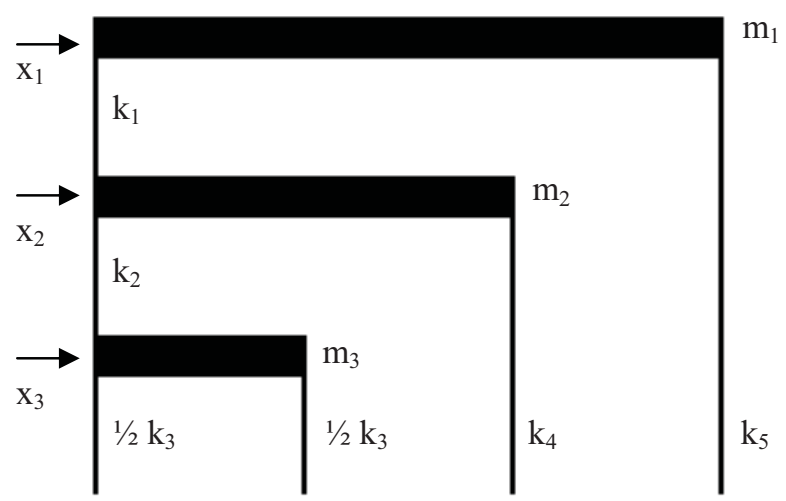

Figure 1. Example shear frame.

$$
\begin{aligned}
& \mathrm{m}_{1}:=0.06 \mathrm{kip} \cdot \frac{\mathrm{sec}^{2}}{\text { in }} \quad \mathrm{m}_{2}:=0.06 \mathrm{kip} \cdot \frac{\mathrm{sec}^{2}}{\text { in }} \\
& \mathrm{m}_{3}:=0.06 \mathrm{kip} \cdot \frac{\mathrm{sec}^{2}}{\text { in }} \quad \text { Masses } \\
& \mathrm{k}_{1}:=10 \cdot \frac{\mathrm{kip}}{\text { in }} \quad \mathrm{k}_{2}:=30 \cdot \frac{\mathrm{kip}}{\text { in }} \quad \mathrm{k}_{3}:=40 \cdot \frac{\mathrm{kip}}{\text { in }} \\
& \mathrm{k}_{4}:=500 \cdot \frac{\mathrm{kip}}{\text { in }} \quad \mathrm{k}_{5}:=10 \cdot \frac{\mathrm{kip}}{\text { in }} \quad \text { Stiffnesses }
\end{aligned}
$$

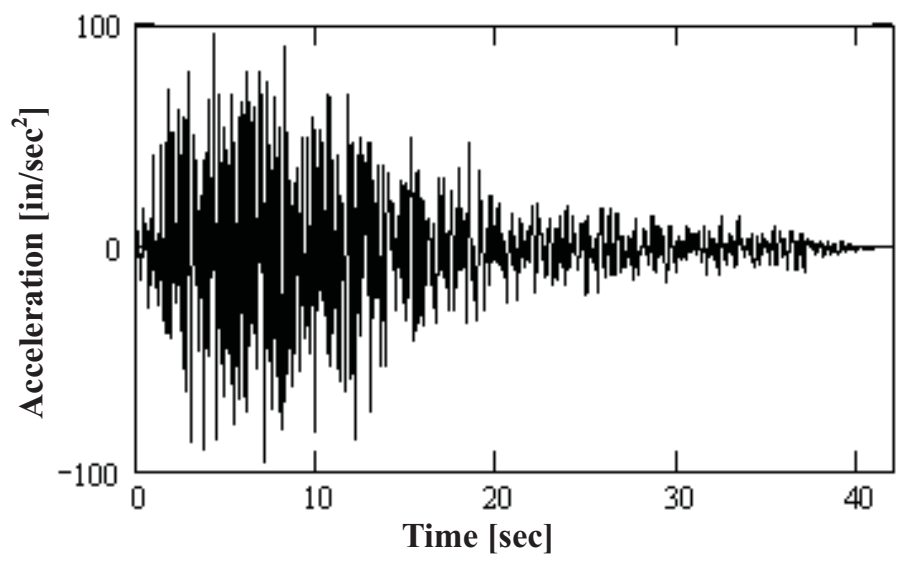

Figure 2. Example earthquake ground motion.

The first step in evaluating the Rayleigh damping coefficients " $\alpha$ " and " $\beta$ " is to calculate the natural frequencies and the corresponding effective mass values for the example shear frame. Below is this portion of the evaluation.

$$
\begin{aligned}
& \mathrm{k}_{\mathrm{o}}:=\left(\begin{array}{ccc}
\mathrm{k}_{1}+\mathrm{k}_{5} & -\mathrm{k}_{1} & 0 \\
-\mathrm{k}_{1} & \mathrm{k}_{1}+\mathrm{k}_{2}+\mathrm{k}_{4} & -\mathrm{k}_{2} \\
0 & -\mathrm{k}_{2} & \mathrm{k}_{2}+\mathrm{k}_{3}
\end{array}\right) \begin{array}{l}
\text { Stiffness } \\
\text { matrix }
\end{array} \\
& \mathrm{m}_{\mathrm{o}}:=\left(\begin{array}{ccc}
\mathrm{m}_{1} & 0 & 0 \\
0 & \mathrm{~m}_{2} & 0 \\
0 & 0 & \mathrm{~m}_{3}
\end{array}\right) \begin{array}{l}
\text { Mass } \\
\text { matrix }
\end{array} \quad \mathrm{I}_{\mathrm{L}}:=\left(\begin{array}{l}
1 \\
1 \\
1
\end{array}\right) \begin{array}{l}
\text { Unit } \\
\text { vector }
\end{array} \\
& \lambda:=\operatorname{sort}\left(\text { eigenvals }\left(\mathrm{m}_{\mathrm{o}}^{-1} \cdot \mathrm{k}_{\mathrm{O}}\right)\right) \quad \text { Sorted eigenvalues } \\
& \lambda=\left(\begin{array}{c}
330 \\
1135 \\
9035
\end{array}\right) \frac{1}{\mathrm{~s}}
\end{aligned}
$$

\section{2}

The United States Government retains, and by accepting the article for publication, the publisher acknowledges that the United States Government retains, a non-exclusive, paid-up, irrevocable, worldwide license to publish or reproduce the published form of this work, or allow others to do so, for United States Government purposes. 
$i:=0 . . \operatorname{last}(\lambda) \quad$ Counting variable

$\left.\Phi^{\prime\langle i}\right\rangle=$ eigenvec $\left(\mathrm{m}_{\mathrm{o}}{ }^{-1} \cdot \mathrm{k}_{\mathrm{O}}, \lambda_{\mathrm{i}}\right) \quad$ Eigenvectors in order

$\Phi^{\prime}=\left(\begin{array}{ccc}1 & 0.013 & -0.019 \\ 0.02 & -0.063 & 0.998 \\ 0.012 & -0.998 & -0.063\end{array}\right)$

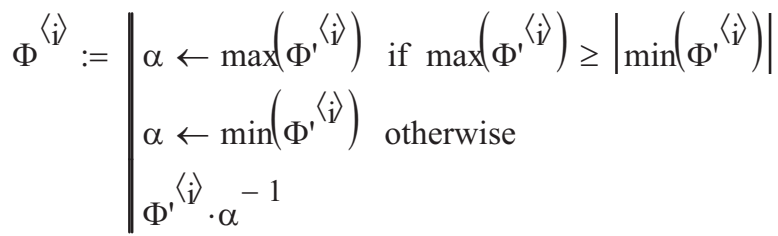

$\Phi=\left(\begin{array}{ccc}1 & -0.013 & -0.019 \\ 0.02 & 0.063 & 1 \\ 0.012 & 1 & -0.064\end{array}\right) \quad$ Normalized eigenvectors

$\omega_{r_{i}}:=\sqrt{\lambda_{i}} \quad \omega_{r}=\left(\begin{array}{l}18.17 \\ 33.69 \\ 95.05\end{array}\right) \frac{\mathrm{rad}}{\mathrm{sec}} \quad \begin{aligned} & \text { Natural frequency } \\ & (\mathrm{rad} / \mathrm{sec})\end{aligned}$

$\mathrm{f}_{\mathrm{r}_{i}}:=\frac{\omega_{\mathrm{r}_{i}}}{2 \cdot \pi} \quad \mathrm{f}_{\mathrm{r}}=\left(\begin{array}{c}2.891 \\ 5.362 \\ 15.128\end{array}\right) \mathrm{Hz} \quad \begin{aligned} & \text { Natural frequency } \\ & (\mathrm{Hz})\end{aligned}$

$\mathrm{m}_{\mathrm{m}}:=\Phi^{\mathrm{T}} \cdot \mathrm{m}_{\mathrm{o}} \cdot \Phi \quad$ Modal mass

$\mathrm{m}_{\mathrm{m}}=\left(\begin{array}{ccc}0.06003 & 0 & 0 \\ 0 & 0.06025 & 0 \\ 0 & 0 & 0.06026\end{array}\right) \mathrm{kip} \cdot \frac{\mathrm{sec}^{2}}{\mathrm{in}}$

$\mathrm{M}_{\mathrm{e}_{\mathrm{i}}}:=\frac{\left(\mid \Phi^{\left.\left\langle{ }_{\mathrm{i}}\right\rangle^{\mathrm{T}} \cdot \mathrm{m}_{\mathrm{O}} \cdot \mathrm{I}_{\mathrm{L}} \mid\right)^{2}}\right.}{\mathrm{m}_{\mathrm{m}_{\mathrm{i}, \mathrm{i}}}} \quad$ Effective mass

$\mathrm{M}_{\mathrm{e}}=\left(\begin{array}{c}0.064 \\ 0.066 \\ 0.05\end{array}\right) \mathrm{kip} \cdot \frac{\mathrm{sec}^{2}}{\mathrm{in}}$

(Note: The absolute value in the effective mass equation is used to turn the $1 \times 1$ array into a scalar value.)

Figure 3 shows the cumulative effective mass ratio versus frequency. Below is the equation used to establish the cumulative effective mass ratio plot data.
$\mathrm{M}_{\text {esum }_{\mathrm{i}}}:=\frac{1}{\mathrm{~m}_{1}+\mathrm{m}_{2}+\mathrm{m}_{3}} \cdot \sum_{\mathrm{j}=0}^{\mathrm{i}} \mathrm{M}_{\mathrm{e}_{\mathrm{j}}} \begin{aligned} & \begin{array}{l}\text { Cumulative } \\ \text { Effective } \\ \text { Mass Ratio }\end{array} \\ & \text { Mass }\end{aligned}$

$\mathrm{M}_{\mathrm{esum}}=\left(\begin{array}{c}0.355 \\ 0.721 \\ 1\end{array}\right)$

A cumulative effective mass ratio plot (as in Figure 3) gives a good indication of where the model response occurs.

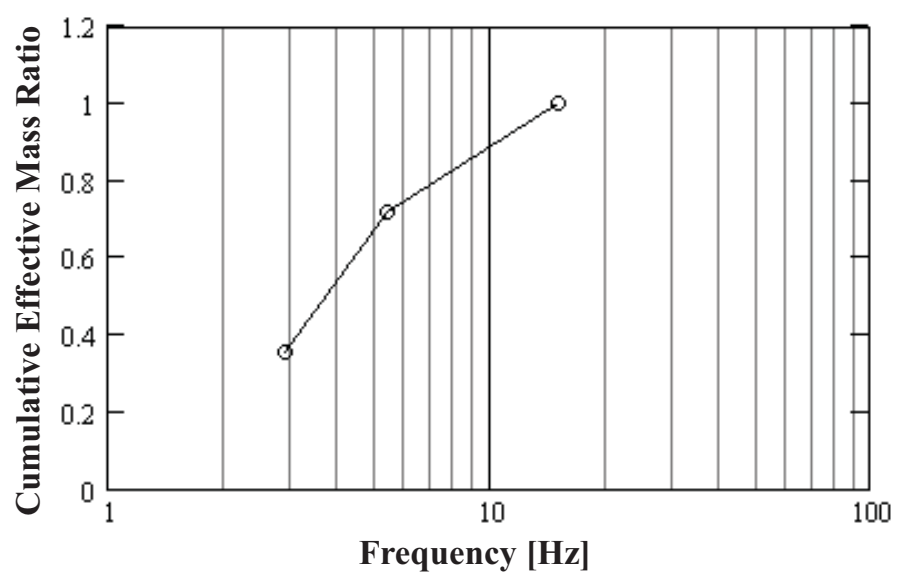

Figure 3. Example cumulative effective mass ratio.

Having natural frequency, the second step is to find the corresponding damped response from the earthquake ground motion (Figure 2). Below is the 5\% damped response established by numerical methods.

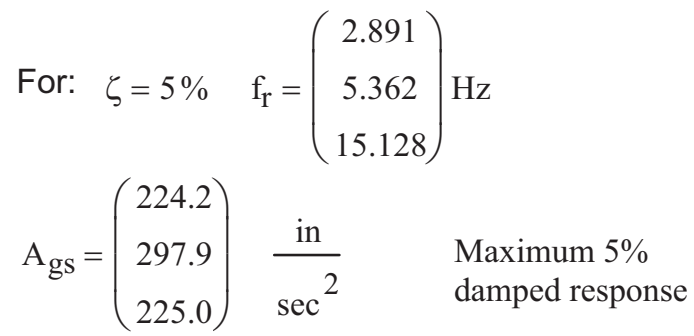

The third step is iterative. For Rayleigh damping, the damping varies with natural frequency (as shown in Figure 4). To control the Rayleigh damping curve, the below derivation is performed. The intent is to force the Rayleigh damping curve to cross the $5 \%$ damping curve at a frequency equal to that of the first point (from Figure 3) that is approximately 0.05 . Because the first point is greater than 0.05 (being 0.355), it is used. For this example, the frequency value is $2.891 \mathrm{~Hz}$. The higher crossing point is varied to achieve a purpose discussed later. For the first iteration, a value is $5 \mathrm{~Hz}$ is selected because it is near the $50 \%$ cumulative effective mass point.

3

The United States Government retains, and by accepting the article for publication, the publisher acknowledges that the United States Government retains, a non-exclusive, paid-up, irrevocable, worldwide license to publish or reproduce the published form of this work, or allow others to do so, for United States Government purposes. 


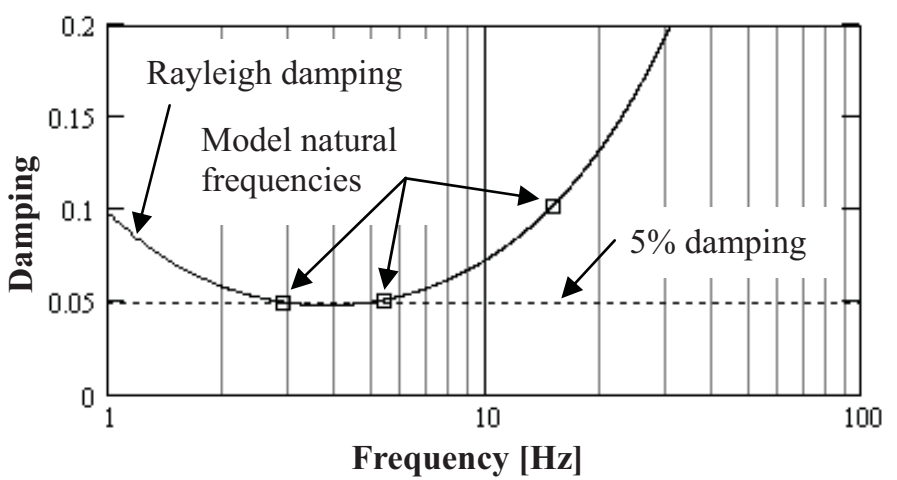

Figure 4. First iteration damping versus frequency.

To establish the Rayleigh damping curve shown in Figure 4 , the following derivation is performed.

$$
\begin{aligned}
& \zeta_{r}(\omega):=\frac{\alpha}{2 \cdot \omega}+\frac{\beta \cdot \omega}{2} \\
& \omega_{0}:=2 \cdot \pi \cdot 2.891 \frac{\mathrm{rad}}{\mathrm{sed}} \\
& \omega_{1}:=2 \cdot \pi \cdot 8.24 \quad \frac{\mathrm{rad}}{\mathrm{sed}} \\
& \zeta:=0.05 \\
& \zeta=\frac{\alpha}{2 \cdot \omega_{0}}+\frac{\beta \cdot \omega_{0}}{2} \\
& \zeta=\frac{\alpha}{2 \cdot \omega_{1}}+\frac{\beta \cdot \omega_{1}}{2} \\
& \left(\begin{array}{cc}
\frac{1}{2 \cdot \omega_{0}} & \frac{\omega_{0}}{2} \\
\frac{1}{2 \cdot \omega_{1}} & \frac{\omega_{1}}{2}
\end{array}\right) \cdot\left(\begin{array}{l}
\alpha \\
\beta
\end{array}\right)=\left(\begin{array}{l}
\zeta \\
\zeta
\end{array}\right) \\
& \left(\begin{array}{l}
\alpha \\
\beta
\end{array}\right):=\left(\begin{array}{cc}
\frac{1}{2 \cdot \omega_{0}} & \frac{\omega_{0}}{2} \\
\frac{1}{2 \cdot \omega_{1}} & \frac{\omega_{1}}{2}
\end{array}\right)^{-1} \cdot\left(\begin{array}{l}
\zeta \\
\zeta
\end{array}\right) \begin{array}{l}
\begin{array}{l}
\text { Rayleigh } \\
\text { damping } \\
\text { coefficients }
\end{array} \\
\text { nam }
\end{array} \\
& \alpha=1.3447 \frac{\mathrm{rad}}{\mathrm{sec}} \\
& \beta=1.4298 \times 10^{-3} \frac{\mathrm{sec}}{\mathrm{rad}}
\end{aligned}
$$

Having the first iteration Rayleigh damping coefficients, the corresponding Rayleigh damped response from the earthquake ground motion (Figure 2) is established. Below is the Rayleigh damped response established by numerical methods.

$$
\zeta_{1_{i}}:=\zeta_{\mathrm{r}}\left(2 \cdot \pi \cdot \mathrm{f}_{\mathrm{r}_{\mathrm{i}}}\right) \quad \begin{aligned}
& \text { First iteration Rayleigh damping values } \\
& \text { for the example frame natural frequencie }
\end{aligned}
$$

For: $\zeta_{1}=\left(\begin{array}{c}5 \\ 5.106 \\ 10.191\end{array}\right) \% \quad \mathrm{f}_{\mathrm{r}}=\left(\begin{array}{c}2.891 \\ 5.362 \\ 15.128\end{array}\right) \mathrm{Hz}$

$$
\mathrm{A}_{\mathrm{gvs}}=\left(\begin{array}{c}
224.2 \\
294.8 \\
184.2
\end{array}\right) \frac{\text { in }}{\mathrm{sec}^{2}} \quad \begin{aligned}
& \text { Maximum Rayleigh } \\
& \text { damped response }
\end{aligned}
$$

A weighting of the difference in maximum Rayleigh damped response and maximum 5\% damped response is now performed with the effective mass. Figure 5 shows the accumulated difference in response scaled by effective mass versus frequency. Below is the equation used to establish the plot points.

$$
\Delta \mathrm{RS}_{\mathrm{wtsum}_{\mathrm{i}}}:=\sum_{\mathrm{j}=0}^{\mathrm{i}}\left(\mathrm{A}_{\mathrm{gvs}_{\mathrm{j}}}-\mathrm{A}_{\mathrm{gs}}\right) \cdot \mathrm{M}_{\mathrm{e}} \mathrm{j}_{\mathrm{j}} \begin{aligned}
& \text { Scaled } \\
& \text { Response } \\
& \text { Difference }
\end{aligned}
$$$$
\Delta \mathrm{RS}_{\mathrm{Wtsum}}=\left(\begin{array}{c}
0 \\
-203 \\
-2251
\end{array}\right) \mathrm{lbf}
$$

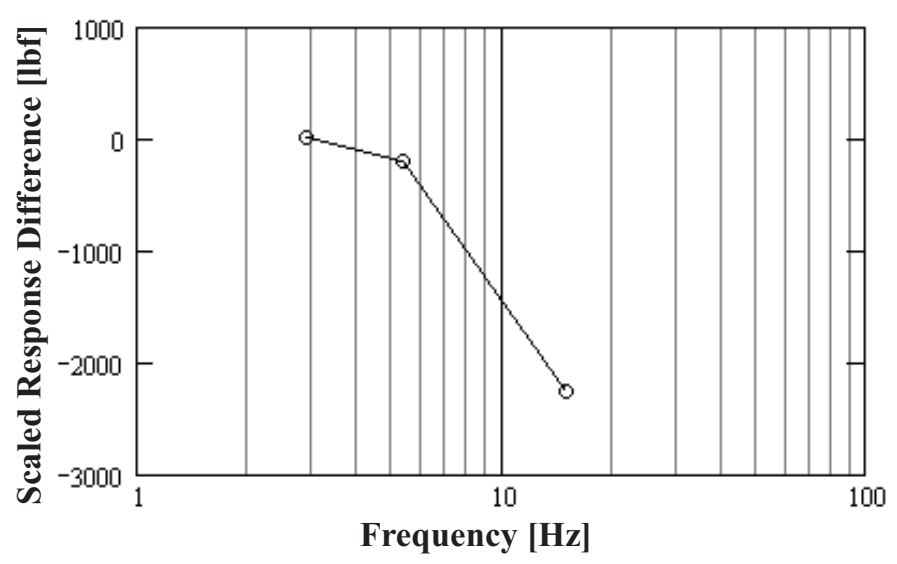

Figure 5. Scaled response difference.

The last point in Figure 5 is the important point. To be acceptable, it must be positive. To optimize it, it should only be slightly positive. Being very negative and relatively substantial (at -2251 lbf), the first iteration Rayleigh damping coefficients are not acceptable. A second iteration must be performed. For

4

The United States Government retains, and by accepting the article for publication, the publisher acknowledges that the United States Government retains, a non-exclusive, paid-up, irrevocable, worldwide license to publish or reproduce the published form of this work, or allow others to do so, for United States Government purposes. 
this iteration, the same calculation is performed except the higher crossing point is set at $8.24 \mathrm{~Hz}$. This produces Raleigh damping constants as shown below which results in the Rayleigh damping curve shown in Figure 6.

$$
\begin{array}{lll}
\alpha=1.3447 \frac{\mathrm{rad}}{\mathrm{sec}} & \begin{array}{l}
\text { Rayleigh } \\
\text { damping } \\
\text { coefficients }
\end{array} \\
\beta=1.4298 \times 10^{-3} & \frac{\mathrm{sec}}{\mathrm{rad}} &
\end{array}
$$

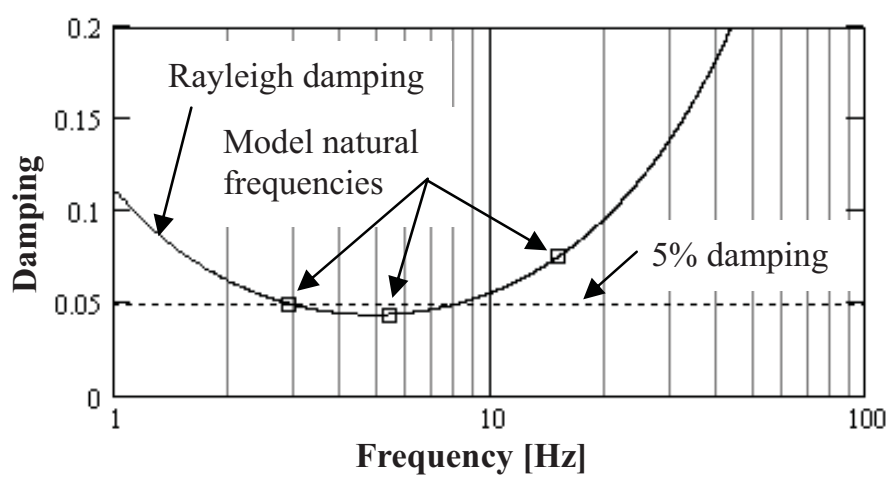

Figure 6. Second iteration damping versus frequency.

The Rayleigh damped response established as shown below.

$$
\begin{aligned}
& \text { For: } \zeta_{1}=\left(\begin{array}{c}
5 \\
4.404 \\
7.503
\end{array}\right) \% \quad \mathrm{f}_{\mathrm{r}}=\left(\begin{array}{c}
2.891 \\
5.362 \\
15.128
\end{array}\right) \mathrm{Hz} \\
& \mathrm{A}_{\mathrm{gvs}}=\left(\begin{array}{c}
224.2 \\
316.1 \\
201.1
\end{array}\right) \frac{\text { in }}{\mathrm{sec}^{2}} \quad \begin{array}{l}
\text { Maximum Rayleigh } \\
\text { damped response }
\end{array}
\end{aligned}
$$

The scaled response difference can then be found numerically and graphically (in Figure 7).

$$
\Delta \mathrm{RS}_{\mathrm{wtsum}}=\left(\begin{array}{c}
0 \\
1203 \\
2
\end{array}\right) \text { lbf } \quad \begin{aligned}
& \text { Scaled Response } \\
& \text { Difference }
\end{aligned}
$$

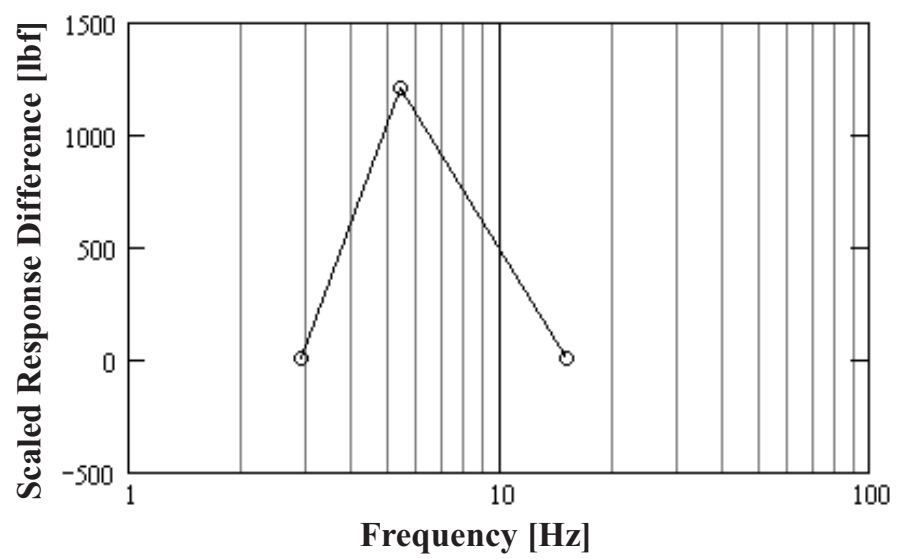

Figure 7. Scaled response difference.

The last point in Figure 7 is positive and relatively insignificant (at $2 \mathrm{lbf}$ ). Consequently, the Rayleigh damping coefficients " $\alpha=1.34 \mathrm{rad} / \mathrm{sec}$ " and " $\beta=0.00143 \mathrm{sec} / \mathrm{rad} "$ represent optimized values for use in time history analysis. For information, Figure 8 shows the modal and Rayleigh damped response spectra. The squares mark the modal damped response for the model natural frequencies. Likewise, the solid circles mark the Rayleigh damped response for the model natural frequencies.

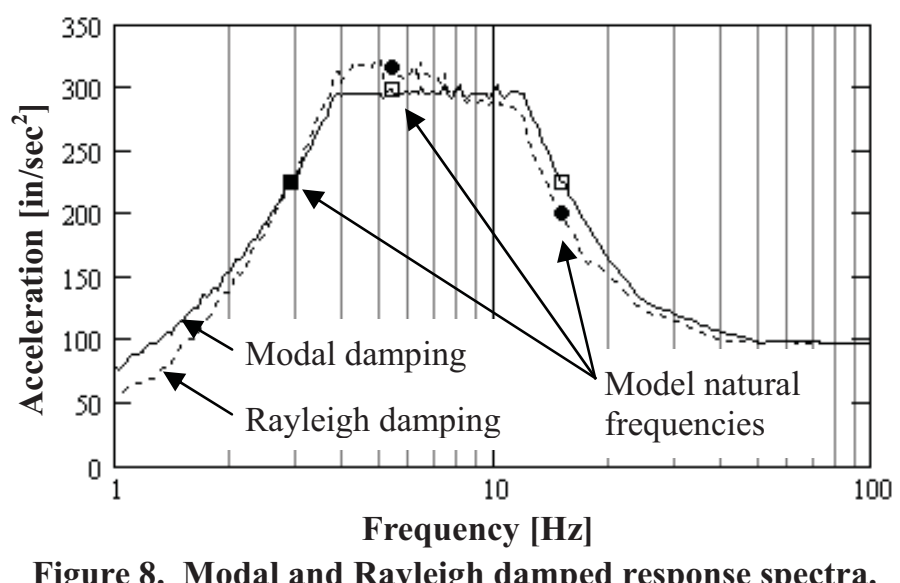

\section{TEST MODEL}

To demonstrate the difference in results, two sets of test model runs are evaluated. The two sets include using modal dynamic analysis with modal damping and using direct integration with Rayleigh damping (having coefficients defined with the proposed approach). Sixteen seismic acceleration time histories (with correlation much less that 30\% between any two) are used to perform the two sets of model runs. (These acceleration time histories are similar to the one shown in Figure 2.)

The test finite element model (shown in Figure 9) is representative of an actual piping system. The model consists of linear beam elements of varying cross section and density

5

The United States Government retains, and by accepting the article for publication, the publisher acknowledges that the United States Government retains, a non-exclusive, paid-up, irrevocable, worldwide license to publish or reproduce the published form of this work, or allow others to do so, for United States Government purposes. 
for the straight pipe and supports. The elbows are parabolic beam elements with cross section properties consistent with flexibility factors defined with the 2007 ASME Section III, Division 1, Subsection NB [4].

The seismic event for this model is run in the z-direction (as identified in Figure 9). Displacement and acceleration results are then established and compared for the pipe nodes (not directly restrained to the ground). Von Mises stresses are also established and compared for the pipe elements. Because it's the differences between modal damped and Rayleigh damped response that is of concern, von Mises stress is considered an acceptable stress value to use. (The actual evaluation of this piping system used the finite element model to generate moments and forces. Von Mises stress is selected for this comparison only because it produced a simple single valued result for comparison.)

Having the model defined, a modal analysis is performed using ABAQUS/Standard [5]. The resulting cumulative effective mass versus frequency plot is shown in Figure 10. This plot indicates the there is significant participation over a wide range for frequencies.

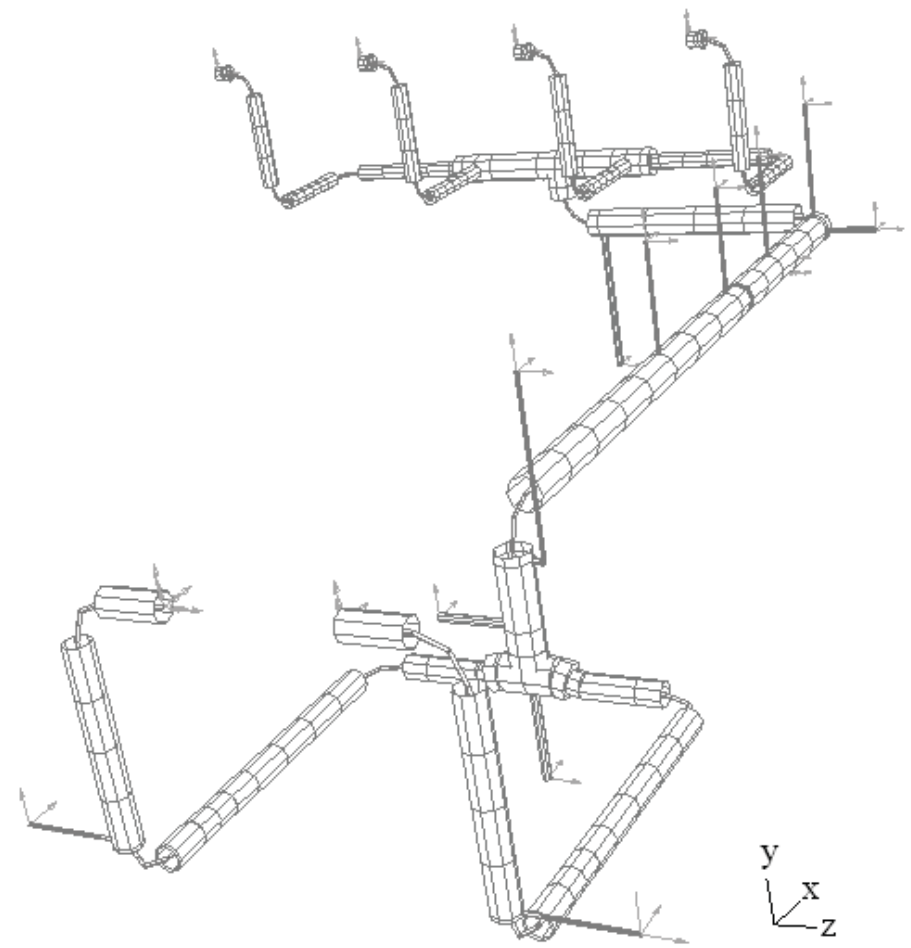

Figure 9. Test finite element model.

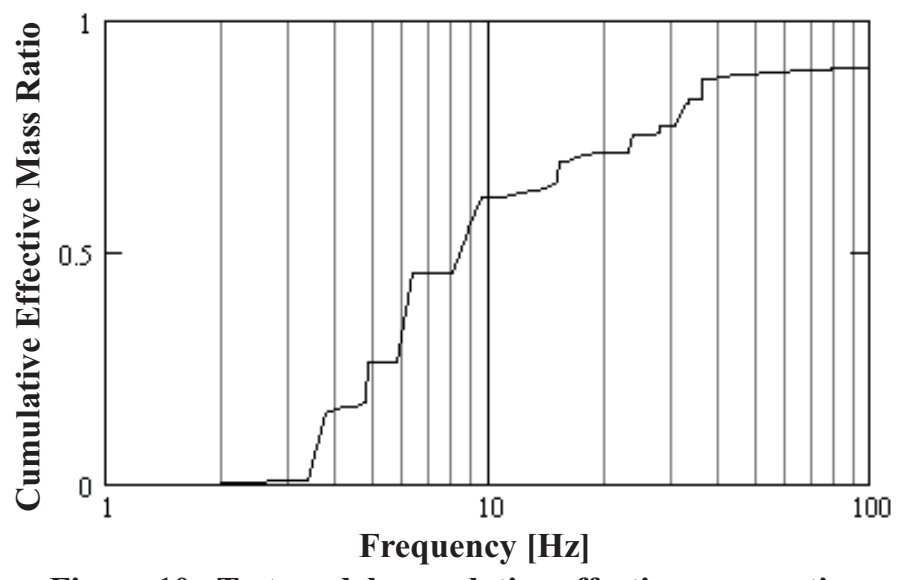

Figure 10. Test model cumulative effective mass ratio.

Considering the cumulative effective mass plot, $90 \%$ of the effective mass is represented for frequencies from $0 \mathrm{~Hz}$ to 100 $\mathrm{Hz}$. This represents a significant missing mass (of 10\%) for modal dynamic analysis. Consequently, the model dynamic model runs are performed using a range of frequencies from 0 $\mathrm{Hz}$ to $1000 \mathrm{~Hz}$. This reduces the missing mass to $2.5 \%$ which is considered to be acceptably low.

For calculating the Rayleigh damping coefficients, considering only the range of frequencies from $0 \mathrm{~Hz}$ to $100 \mathrm{~Hz}$ is sufficient. This is because the high frequency response (for this example) is negligibly different between the modal damped and Rayleigh damped response spectra (as demonstrated in Figure 13).

Optimizing the Rayleigh damping coefficients for each model run produces the sixteen scaled response difference curves shown in Figure 11. This results in the damping and response spectra shown in Figures 12 and 13.

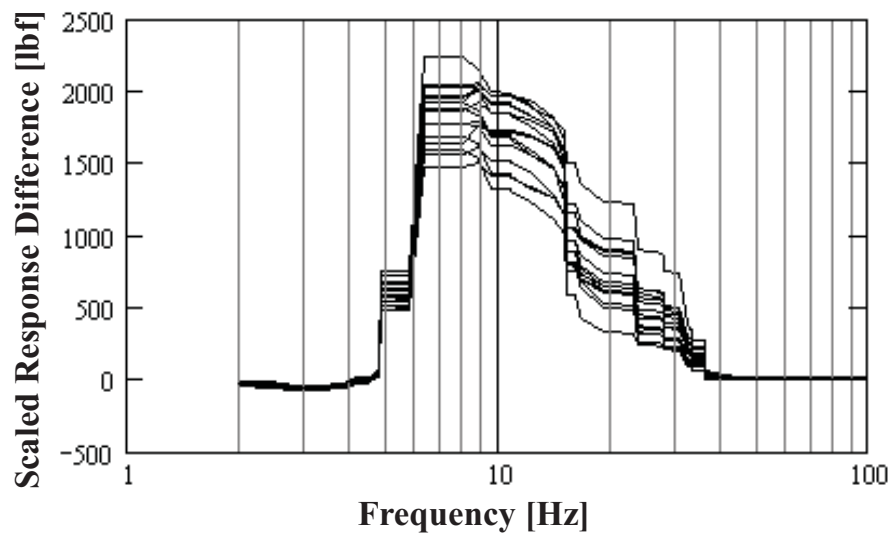

Figure 11. Sixteen scaled response difference curves.

6

The United States Government retains, and by accepting the article for publication, the publisher acknowledges that the United States Government retains, a non-exclusive, paid-up, irrevocable, worldwide license to publish or reproduce the published form of this work, or allow others to do so, for United States Government purposes. 


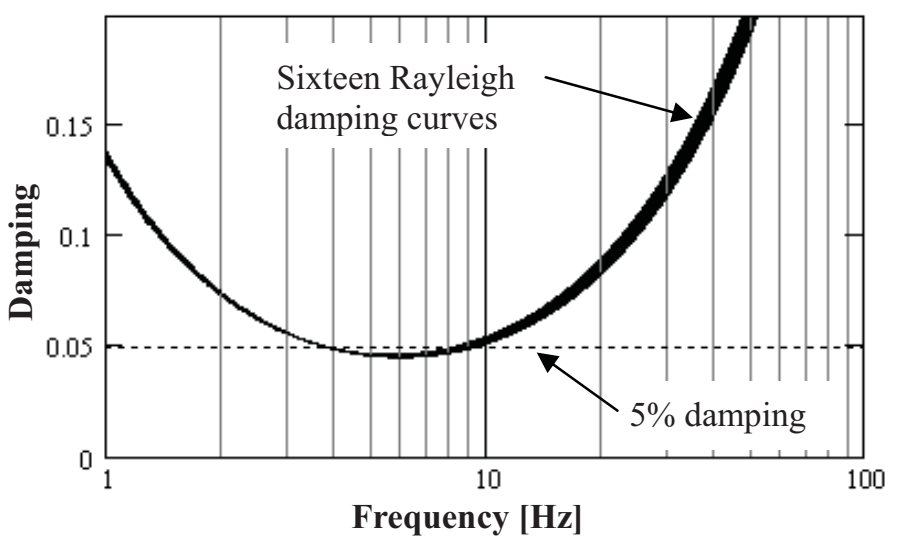

Figure 12. Sixteen damping versus frequency curves.

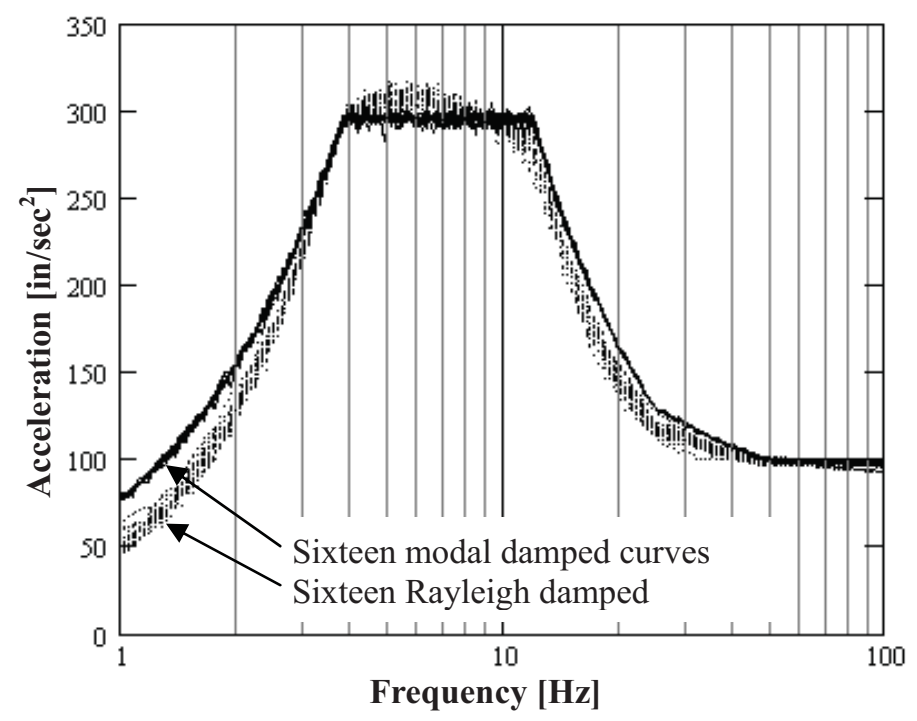

Figure 13. Sixteen pairs of test model response spectra.

Having the optimized Rayleigh damping coefficients, the sixteen models are run for the direct integration with Rayleigh damping and for the corresponding modal dynamic with 5\% damping. (The models were run using ABAQUS/Standard [5] which outputs total motion for the direct integration solution and relative motion for the modal dynamic solution. For comparison, the direct integration total displacement was converted to relative displacement. Likewise, the modal dynamic relative acceleration was converted to total acceleration.)

For comparison between the Rayleigh damped and modal damped response, Figures $14-16$ are generated. The data points for fractional change in each are based on the Rayleigh damped peak response minus the modal damped peak response divided by the modal damped peak response. Consequently, positive values indicate that the Rayleigh damping model produces a higher peak response. The other axis on the figures is the modal damped peak response. This spreads the data points out relative to the magnitude of the peak response where they occur. (Figure 14 has data points with modal damped peak response values near zero that are off of the plot in the positive direction. These were considered insignificant due to them representing insignificant response.)

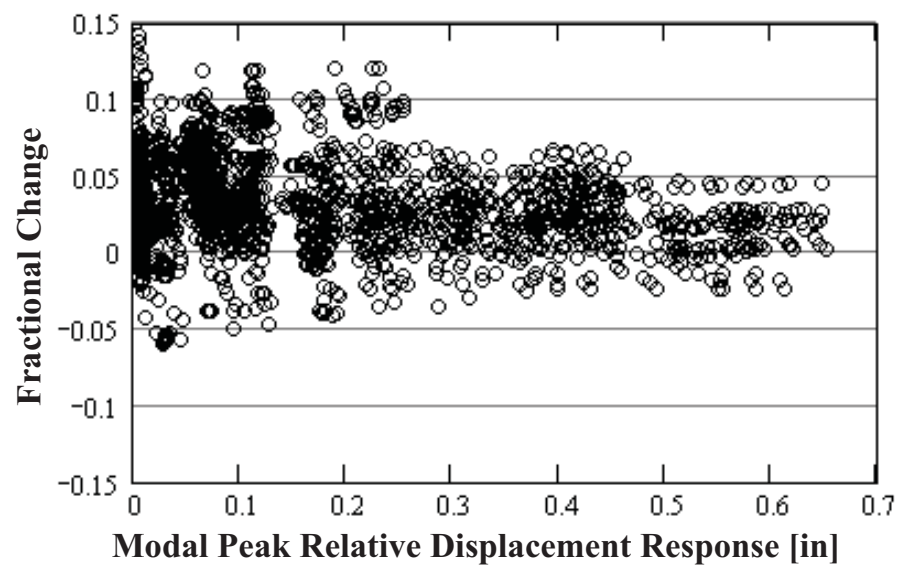

Figure 14. Rayleigh and modal displacement comparison.

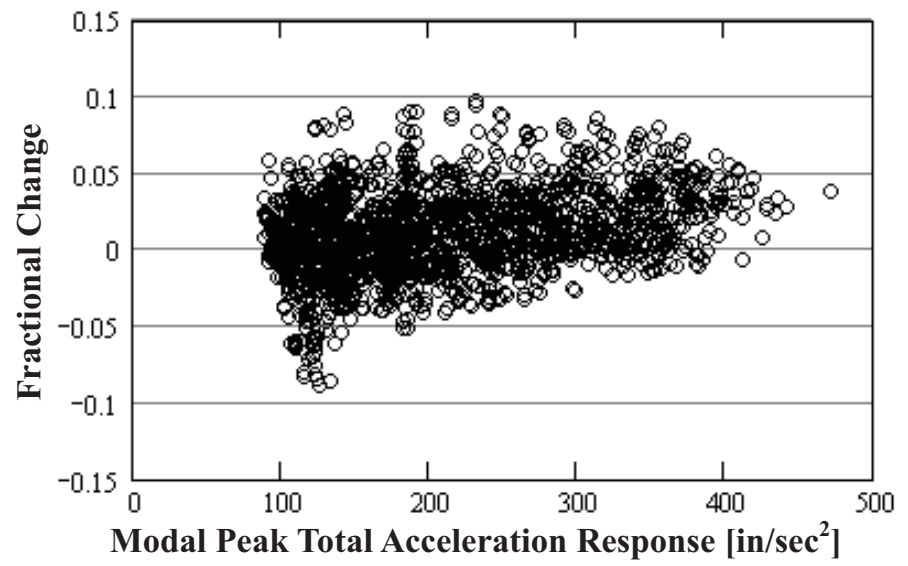

Figure 15. Rayleigh and modal acceleration comparison.

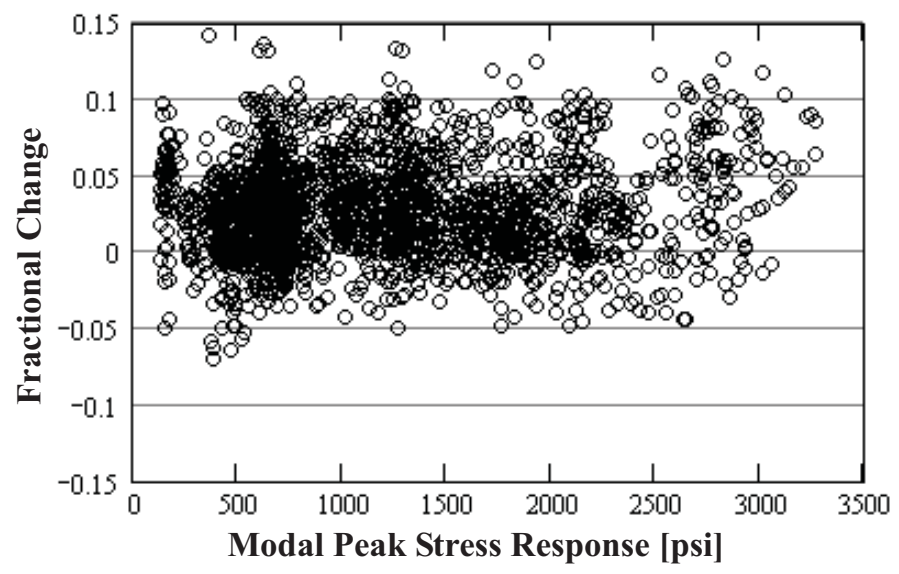

Figure 16. Rayleigh and modal stress comparison.

A $2.5 \%$ shift upward in the comparison results can be expected due to the $2.5 \%$ missing mass in the modal dynamic solution. Also, the higher 5\% crossing point for the Rayleigh 7

The United States Government retains, and by accepting the article for publication, the publisher acknowledges that the United States Government retains, a non-exclusive, paid-up, irrevocable, worldwide license to publish or reproduce the published form of this work, or allow others to do so, for United States Government purposes. 
damping curve was optimized to the nearest $0.01 \mathrm{~Hz}$ that caused the scaled response difference to end positive. This also causes a slight upward shift.

For additional information, Figures $17-19$ show a comparison of the maximum peak modal response at a location to the minimum peak modal response at that location. The data points for modal fractional change in each are based on the maximum peak modal response minus the minimum peak modal response divided by the maximum peak modal response.

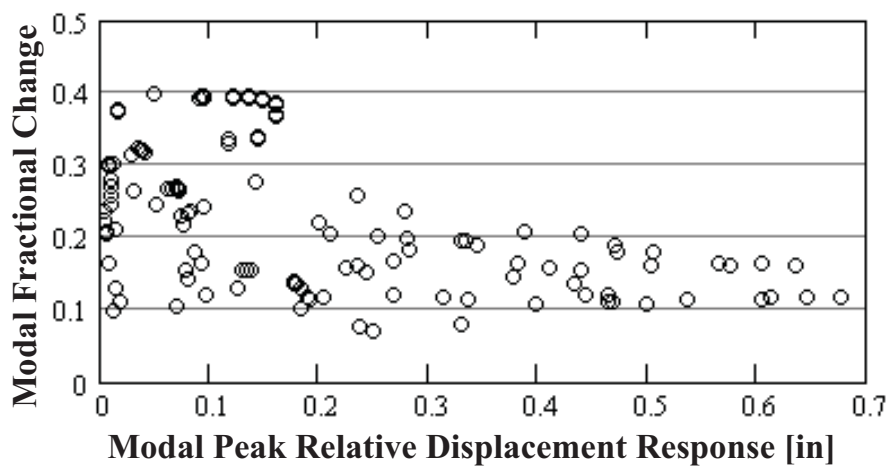

Figure 17. Extreme modal displacement comparison.

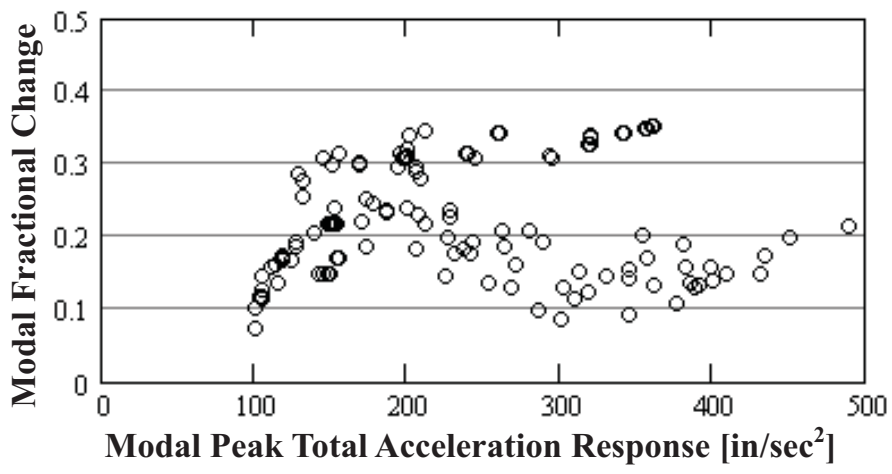

Figure 18. Extreme modal acceleration comparison.

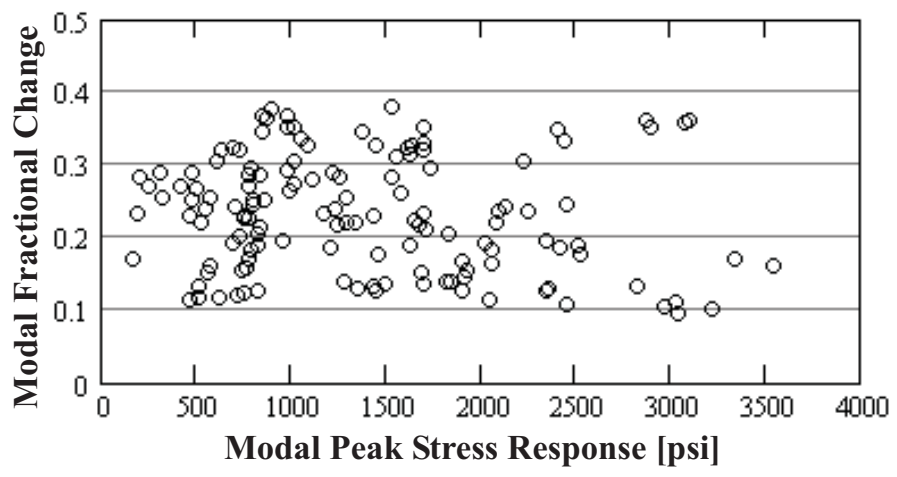

Figure 19. Extreme modal stress comparison.

For this test problem, Figures 17 - 19 demonstrate more substantial scatter than Figures $14-16$. This indicates that there is more scatter caused by the different seismic acceleration time histories than caused by the difference between Raleigh and modal damping for a given seismic acceleration time history.

More substantial difference between Raleigh and modal damping could be expected if the cumulative effective mass in Figure 10 were shifted to lower frequencies. Evaluation of response spectra as in Figure 13 should be performed as an indication of the scale of the difference. Considering Figure 13, the more substantial the difference is between the modal damped and Rayleigh damped curves (above the lowest significant natural frequency), the more substantial the difference in the results.

\section{CONCLUSION}

Nonlinearities, whether geometric or material, need to be addressed in seismic analysis. This may motivate an analyst to evaluate the seismic response using direct time integration with Rayleigh damping. An approach has been proposed for selection of Rayleigh damping coefficients to be used in seismic analyses to produce response that is consistent with Modal damping response. The approach uses the difference between the modal damping response and the Rayleigh damping response along with effective mass properties of the model being evaluated to match overall system response levels. An example problem is included for clarification of the process. Also, a test problem is performed to demonstrate the difference in the results that can be expected in an actual problem. Ultimately, the analyst must ensure that this approach is reasonable for the given problem. The approach, however, should be reasonable for a wide range of problems.

\section{ACKNOWLEDGEMENTS}

This manuscript has been authored by Battelle Energy Alliance, LLC under Contract No. DE-AC07-05ID14517 with the U.S. Department of Energy. The United States Government retains and the publisher, by accepting the article for publication, acknowledges that the United States Government retains a nonexclusive, paid-up, irrevocable, world-wide license to publish or reproduce the published form of this manuscript, or allow others to do so, for United States Government purposes.

The work described in this paper was performed as part of the Advanced Test Reactor Life Extension Project.

\section{REFERENCES}

[1] ASCE 4-98, Seismic Analysis of Safety-Related Nuclear Structures and Commentary, American Society of Civil Engineers, 1998.

[2] ASCE/SEI 43-05, Seismic Design Criteria for Structures, Systems, and Components in Nuclear Facilities, 2005.

[3] Mathcad 14.0, Parametric Technology Corporation, 2007.

[4] 2007 American Society of Mechanic Engineers (ASME) Boiler \& Pressure Vessel Code BPVC, Section III, Division 1 Subsection NB, "Class 1 Components," ASME International. [5] ABAQUS Standard, Version 6.7-5, ABAQUS Inc., 2007.

8

The United States Government retains, and by accepting the article for publication, the publisher acknowledges that the United States Government retains, a non-exclusive, paid-up, irrevocable, worldwide license to publish or reproduce the published form of this work, or allow others to do so, for United States Government purposes. 